\title{
Hybrid Solar And Wind Energy Potential Map of Turkey and Cost Analysis of 1 MW Hybrid Power Plants
}

\author{
Furkan SENGULER ${ }^{1}$ and Zehra YUMURTACI ${ }^{1}$ \\ ${ }^{1}$ Energy Subdivision, Department of Mechanical Engineering, \\ Y1ldız Technical University, \\ İstanbul, Turkey
}

\begin{abstract}
While the need for electrical energy has increased over time, a renewable energy source alone cannot meet this increase yet. In the future, alternative ways of decreasing loyalty to fossil fuels are sought. Hybrid energy is an important step in this regard. Hybrid energy is a good alternative to fossil fuels, due to the high production capacity compared to solar or wind energy alone. In Turkey, investment of solar and wind energy increasing day by day. While solar and wind power getting crucial for Turkey it is important to know how much potential of hybrid energy exists in the country with a map. Purpose of this study is by combining solar and wind potential of Turkey, creating the hybrid energy potential map. In addition, with considering the economic conditions the establishment of a hybrid power plant scenario created. This scenario is aimed to determine whether the hybrid power plant feasible or not. In this regard, solar and wind potential in 81 provinces were determined. By combining wind and solar potential data at the same points, hybrid energy potentials were determined. Then, if a 1MW power plant was established for each province based on the determined hybrid potential data, the cost analysis of the value of the investment was made based on the appropriate economic data. According to the results of the study especially in Hatay in Turkey were found to be quite high wind potential and solar potential at an adequate level. Also, provinces such as Çanakkale and Karaman follow Hatay as high yielding regions. After the studies, Turkey has been found to be a suitable place for hybrid energy. The hybrid power plant, which uses clean and renewable energy sources can reduce fossil fuel utilization rate. So hybrid energy can be an alternative way to save both Turkey and the World.
\end{abstract}

Key Words: Wind, Solar, Turkey, Hybrid Potential Map, Cost Analysis

\section{INTRODUCTION}

While population of Turkey increases, the demand of Energy increases parallelly. In Turkey great amount of energy generation depends on fossil fuels. In 2018 Turkey generated its energy from different energy sources. 303.9 billion kWh energy generated in 2018. These energy sources were; $37.3 \%$ coal, $29.8 \%$ natural gas, $19.8 \%$ hydraulic energy, $6.6 \%$ wind energy, $2.6 \%$ solar energy, $2.5 \%$ geothermal energy and $1.4 \%$ energy generated by using other sources. Also there is 7423 power plant in Turkey at the end of the 2018. There are 653 hydroelectric power plant, 42 coal power plant, 249 wind power plant, 48 geothermal power plant, 320 natural gas power plant, 5868 solar power plant and 243 other sourced power plant in Turkey [1].

It is known that fossil fuels will be run out at the future. Also fossil fuels are causing global warming and polluting the air. On the other hand there is not enough fossil fuel resources in Turkey. Dependence of fossil fuel is economic burden for Turkey. While dependence of fossil fuel is continuing, portion of energy generation of fossil fuels can be decrease by using alternative energy. For Turkey it is great alternative to use solar and wind energy according the potential that country have [2,3].

Wind energy can be generated both onshore and offshore and it is clean and renewable form of energy. It has not need any fuel and not emits carbon dioxide. Wind energy generated by different temperature level caused from solar radiation. This creates change in pressure levels and different pressure levels create movement in the air. 2\% of solar energy that reach to Earth is transforming wind energy. Wind turbine is most important structure that generate electric energy from wind energy. Wind turbine extract energy from wind by using its blades. While wind rotates the turbine blades, the electric is generated from turning shaft that connected with blades. Kinetic energy transforms into to electric energy and then transferred to grid network. 
In Turkey wind energy potential is predicted as $48000 \mathrm{MW}$. The area that correspond the potential is about equal to $1.3 \%$ of Turkey's area. In 2018, 19.882 billion kWh electric generated from wind energy. And total installed capacity is 7005 MW for Turkey [4].

Solar energy generated by using sun is an energy type which can be transformed into electric energy or thermal energy. Solar energy can be generated by using different methods. The most popular method is using photovoltaic solar panel. Photovoltaics directly convert solar energy into electricity. They work on the principle of the photovoltaic effect. Photovoltaic panels working when certain materials are exposed to light. They absorb photons and release free electrons which called as the photoelectric effect. Photovoltaic effect is a method of producing direct current electricity based on the principle of the photoelectric effect. Based on the principle of photovoltaic effect, solar cells or photovoltaic cells are made. They convert sunlight into direct current (DC) electricity. But, for most of the times AC power required and hence, solar power system consists of an inverter.

Solar energy is clean, cheap and renewable energy source. So solar energy does not pollute environment. At each point that sunlight reaches to Earth solar energy can be generated. Since the solar energy source is sun, it has infinite source. Solar energy can be used for heating water, power homes and building, power cars. Also it can be use as power plant.

At the end of 2018 there are 5863 solar power plant in Turkey. Total installed solar power plant capacity is 5063 MW. 7477.3 GWh of electric energy generating from solar energy in Turkey. This correspond to $2.5 \%$ of total energy generation $[5,6]$.

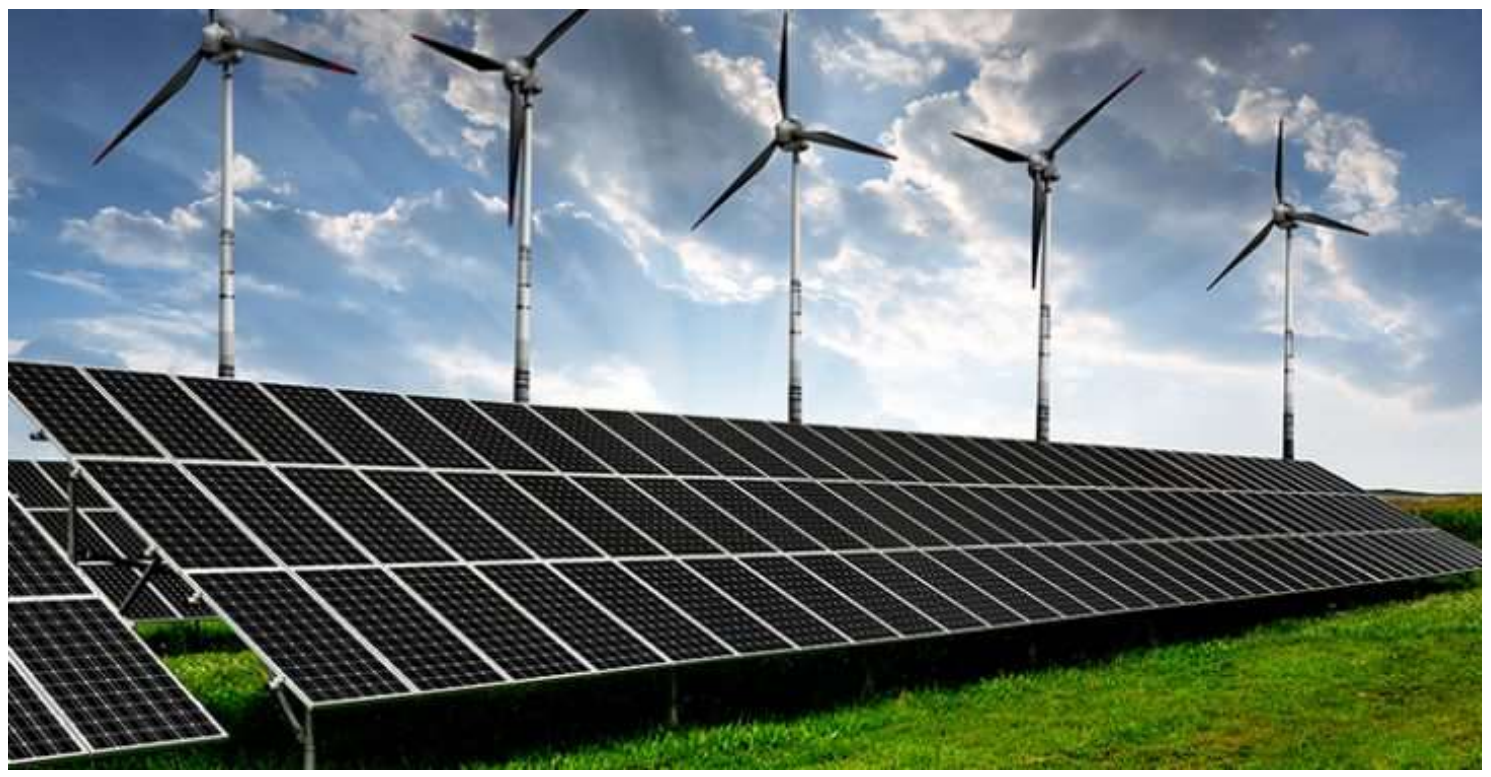

Figure 1.1: Hybrid Power Plant

Wind and solar energy not only prevent running out of fossil fuels. Also generate clean energy and prevent the environment pollution. But renewable energy sources are not continuous. Continuity problem is one of the biggest issue for clean and renewable energy. When there is energy demand there have to be energy to supply. But solar energy is only available at the daytime and wind energy is available at the certain times. So renewable energy sources are not predictable. This situation creates continuity issue. Because of that renewable energy source must be continuous to correspond the energy demand. This problem can be solved by using integrated energy sources. By using both solar and wind energy together energy supply can be more continuous and generates more power. Also renewable energy sources may not be predictable but some crucial information that important for generating energy such as wind speed and solar radiation can be determined [7-11]. The technology that using at production of wind turbine and photovoltaic panel, crated new possibilities for generating electric energy from wind and solar energy [12]. Renewable energy sources are very applicable alternative at rural areas. At locations that have not great renewable energy potential using hybrid solar and wind energy together is more efficient instead of using only solar or wind energy have found out according to studies [13]. Using battery system as attachment to hybrid system increases the continuity of energy supply $[14,15]$. Generally wind-solar hybrid power plant consists wind turbine, photovoltaic solar panel, controller and storage unit. Electric generated from wind turbine is not stabile. So some control units and invertors made this electric energy consistent and storable. Than stored energy can be used for local or other purposes. In photovoltaic order, solar panels can be align as series or parallel. Generated electric energy can be stored and used as alternative (AC) or direct current (DC). Hybrid systems have high energy generation capacity. Also electric generation cost and operation, maintenance cost is low [16]. In this study by considering wind and solar hybrid energy, Turkey's potential has been determined. The main reason for choosing the wind and solar hybrid system is the advantages of the hybrid system. Hybrid energy, which is one of the solutions reached as a result of the desire for a more livable future, is aimed to be more widespread in Turkey and to benefit both the economy and the environment. 


\section{METHODOLOGY}

\subsection{Wind and Solar Potential}

Wind and potential maps of Turkey has been examined. After the examination best possible wind speed and solar radiation located for each 81 provinces and 7 region of Turkey. According to figure 2.1 and figure 2.2 wind potential has been determined. But this determination has been made by correspond to figure 2.3. For all the provinces sufficient point is selected for both wind and solar energy potential. To combine solar and wind energy potential for creating the hybrid energy potential this path has been followed;

1. Determining the highest solar radiation zone for province

2. Determining the highest wind speed zone for province

3. Comparison between highest solar radiation and wind speed zone

4. If these zones are at same location taking the data from both maps

5. If these zones are not at the same location detailed examination of province

6. Choosing the highest potential position by letting minimum reduction of wind or solar potential and taking the data

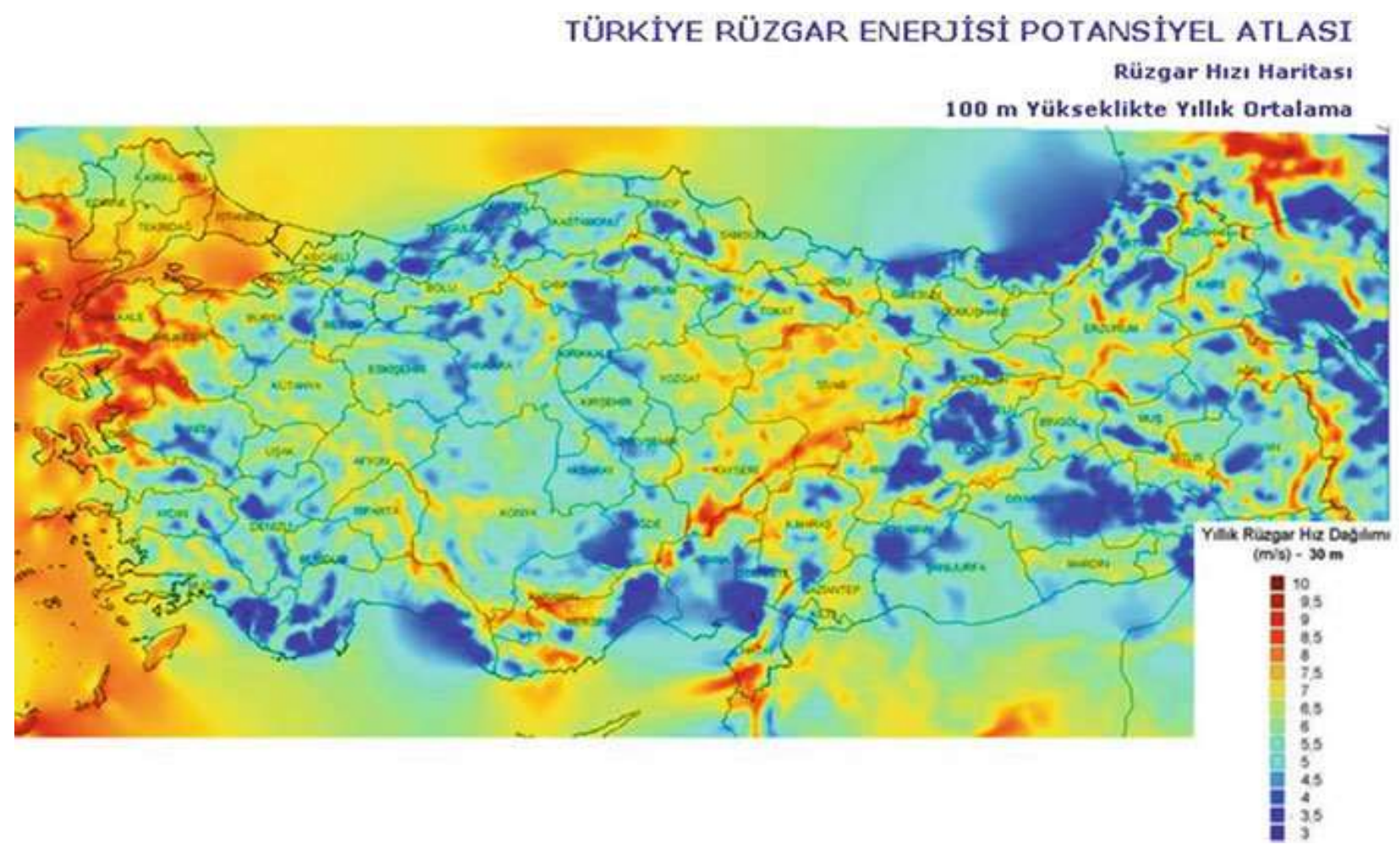

Figure 2.1: Wind Speed Data for Turkey [17]

From figure 2.1 yearly average wind speed has been read which one of the important aspect for the wind energy. Wind speed data has been shown by using colors. The unit for the data shown as $\mathrm{m} / \mathrm{s}$. As seen on map at the west side of country there is great wind energy potential. Also at Center Anatolian Region, south side and some eastern Anatolian zone good wind potential can be detected. Those wind speed data shows at 100 meter high from ground yearly average speeds. When considering wind energy potential capacity factor is another important aspect for the calculating wind energy. At the below capacity factor data has been shown in figure 2.2. It is similar with figure 2.1 but at some point colors are differing and data shown is using percent. This data is crucial for calculating yearly total energy which is needed for determining the wind potential and cost analysis. 

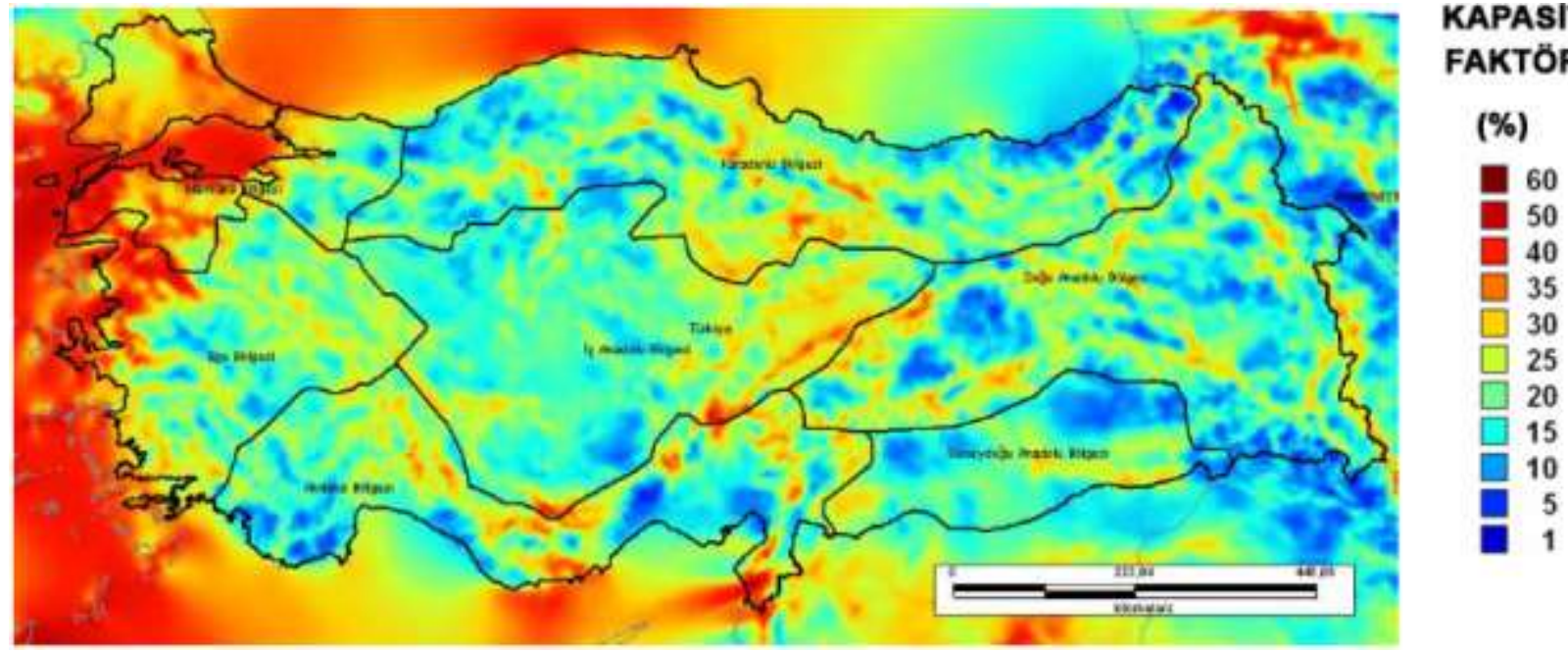

Figure 2.2: Wind Capacity Factor Data for Turkey [17]

After collecting the data calculation become possible. But still some selection has to be made to calculate the wind energy potential such as turbine blade diameter, air density, and losses. Power has been calculated from using (2.1)

$$
\mathrm{P}=\frac{1}{2} * \rho * \mathrm{v}^{3} * \mathrm{~A} * \mathrm{n}_{\mathrm{b}} * \mathrm{n}_{\mathrm{l}}
$$

Turbine blade diameter $\left(44 \mathrm{~m}\right.$ ) is selected. It is using to calculate are that blades span. The aerodynamic losses $\mathrm{n}_{1}$ is selected as $50 \%$ of betz limit. The other selected parameters are $\mathrm{n}_{\mathrm{b}}$ is betz limit which is $59.3 \%, \rho$ is air density which is $\left(1.23 \mathrm{~kg} / \mathrm{m}^{3}\right)$, Wind speed is taken from the Figure 2.1.

$$
\mathrm{E}=\mathrm{P} * \mathrm{cf} * 365 * 24 / 1000
$$

(2.2) is used for calculating the total wind energy. $\mathrm{P}$ is power as Watt, $\mathrm{cf}$ is capacity factor value which taken from Figure 2.2. 24 hour and 365 days considered for yearly calculation. Then it is divided by 1000 to find out kWh per yearly energy generation.

In the figure 2.3 Turkey's solar radiation data has been given. The color red to blue represent the intensity of solar radiation. Where red color indicates high solar radiation, blue color indicates low solar radiation. As seen from the figure 2.3 The North side of Turkey has less solar radiation especially at the north east. The south of Turkey has much higher potential than north side. Southwest side has the most solar radiation according to data given by Turkish State Meteorological Service.

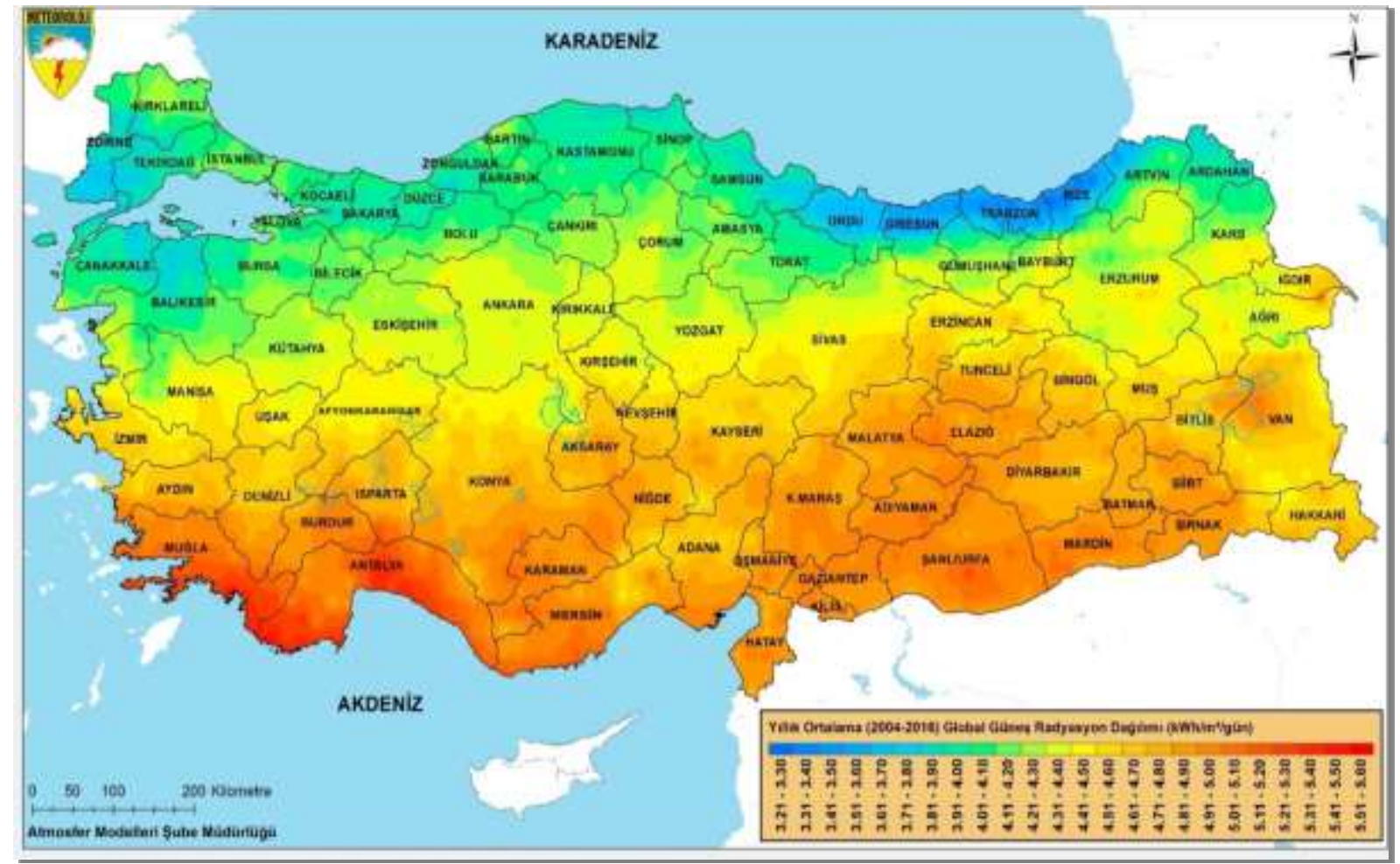

Figure 2.3: Solar Radiation Speed Data for Turkey [18] 
The unit of data shown in the map is $\mathrm{kWh} / \mathrm{m}^{2} /$ day. This data gathered by Turkish State Meteorological Service which shows yearly average value between 2004 and 2016. To calculate the yearly solar energy generation (2) has used.

$$
\mathrm{E}=\mathrm{A} * \mathrm{r} * \mathrm{H} * 365 * \mathrm{PR}
$$

Where A is area of photovoltaic solar panel which is selected $\left(1,9625 \mathrm{~m}^{2}\right), \mathrm{E}$ is yearly energy generation $(\mathrm{kWh}), \mathrm{r}$ is chosen solar energy efficiency $(18.5 \%), \mathrm{H}$ is yearly average radiation $\left(\mathrm{kWh} / \mathrm{m}^{2}\right), \mathrm{PR}$ is performance ratio which selected as 0.75 . Yearly solar energy potential has calculated after this work.

After the calculations to compare with wind and solar data, calculated values have divided to area for wind turbine and photovoltaic solar panel. Since 1 wind turbine generates more energy than 1 photovoltaic solar panel. Wind energy divided to turbine blade span area and Photovoltaic solar panel divided to panel area. So they can be compared to each other as percentage of total wind and solar potential.

\subsection{Cost Analysis}

To calculating the cost analysis some parameters have been chosen. Cost analysis calculation based on 1MW hybrid power plant for each provinces. Installing power portion for wind and solar energy determined by using calculated wind and solar energy percentages. For wind energy $200 \mathrm{~kW}$ Turbine and for solar energy $250 \mathrm{~W}$ photovoltaic panel selected. Also storage system cost has been added to cost analysis.

The price for wind turbine is taken as $280000 \$$, for photovoltaic panel $84 \$$ and for the storage system $95000 \$$. Turbine and solar panel count varies according to provinces. So price can change for different cities.

As the operation and maintenance cost, for wind turbine it is taken as $2 \%$ of investment cost. Escalation of operation and maintenance cost is selected as 1\%. For solar panel it is taken as $1 \%$ of investment cost. Escalation of operation and maintenance cost is selected as $1 \%$ same with wind turbine [19]. After calculating the first year O\&M cost it is calculated for 25 years which is lifetime of system.

There are some governmental incentives for renewable energy in Turkey. According to regulations first 10 years wind energy selling price is set to $0.073 \$ / \mathrm{kWh}$. For solar energy this price is $0.133 \$ / \mathrm{kWh}$ [20]. After 10 years selling price returns its normal values. Normal selling price is $0.06 \$ / \mathrm{kWh}[21]$. But this price will be applied after 10 years. Because of that $2 \%$ of yearly electricity price increase applied to normal price until the 11 th year.

$$
\mathrm{F}_{10}=1 \text { st year electricity cost } *(\mathrm{~F} / \mathrm{P}, \% 2,11)=11 \text { th year electricity price } \$ / \mathrm{kWh}
$$

After this calculation 1st and 11th year revenue has been calculated by using yearly electric generation which calculated previously. When taking into account the turbine and solar panel numbers yearly total revenue has been calculated. First 10 year constant revenue and last 15 years from normal price to increasing economic model has been constructed. Both wind energy revenue and solar energy revenue combined together to finding out total hybrid energy system net present worth. Rate of discount taken as $18.5 \%$ and lifetime of the system chosen as 25 years. Bpw can be considered as revenue and Cpw as cost.

$$
\mathrm{Bpw}=1 \text { st year revenue } *(\mathrm{P} / \mathrm{A}, \% 18.5,10)+11 \text { th year revenue* }(\mathrm{P} / \mathrm{A} 1, \% 2, \% 18.5,15) *(\mathrm{P} / \mathrm{F}, \% 18.5,10) \quad(5)
$$

$\mathrm{Cpw}=$ Investment Cost $($ Solar + Wind + Storage $)+1$ st year wind turbines O\&M cost*(P/A1, \%1, \% 18.5, 25) + 1st year solar panels $\mathrm{O} \& \mathrm{M}$ cost *(P/A1, \%1, \%18.5, 25)

$$
\mathrm{Npw}=\mathrm{Bpw}-\mathrm{Cpw}
$$

If the Npw value bigger than 0 then the investment considered as feasible. If the Npw value is lower than 0 investment considered as unprofitable. Also unit electric energy cost is calculated for each provinces. To calculate it investment cost has been divided to yearly cost and rate of discount has used in the formula (10).

$$
\mathrm{g}_{\mathrm{k}}=\text { Investment cost } * 0.185 *(1+0.185) 25 /((1+0.185) 25-1) / \text { Yearly generated energy }
$$

$$
\mathrm{g}_{\mathrm{m}}=\text { Yearly Maintenance Cost/ Yearly generated energy }
$$

$$
\mathrm{g}=\mathrm{g}_{\mathrm{k}}+\mathrm{g}_{\mathrm{m}}(\$ / \mathrm{kWh})
$$

\section{RESULTS AND DISCUSSION}

\subsection{Hybrid Potential Data}

The calculation has been made by using (1), (2) and (3). After the calculations wind potential and solar potential has been found. First of all yearly energy potential has been found and then it is divided to area $\left(\mathrm{m}^{2}\right)$ of turbine and solar panel. Wind and solar potential percentage is calculated by using total wind and solar potential. Wind and solar energy potential given as yearly based. At the right side of the table 2.1 Yearly total energy is given for 1 turbine and 1 solar panel. 
Table 2.1. Wind and Solar Energy calculation for Marmara Region

\begin{tabular}{|c|c|c|c|c|c|c|}
\hline Province & $\begin{array}{c}\text { Wind } \\
\text { potential } \\
\left(\mathbf{k W h} / \mathbf{m}^{2}\right) \\
\end{array}$ & $\begin{array}{c}\text { Solar } \\
\text { potential } \\
\left(\mathbf{k W h} / \mathbf{m}^{2}\right) \\
\end{array}$ & $\begin{array}{c}\text { Wind } \\
\text { percentage }\end{array}$ & $\begin{array}{c}\text { Solar } \\
\text { percentage }\end{array}$ & $\begin{array}{c}\text { Yearly wind } \\
\text { energy } \\
\text { (kWh/turbine) }\end{array}$ & $\begin{array}{c}\text { Yearly solar } \\
\text { energy } \\
(\mathrm{kWh} / \text { panel })\end{array}$ \\
\hline İstanbul & 258.93 & 210.42 & $55.2 \%$ & $44.8 \%$ & 393703.82 & 412.96 \\
\hline Edirne & 356.96 & 192.70 & $64.9 \%$ & $35.1 \%$ & 542773.09 & 378.17 \\
\hline Tekirdağ & 356.96 & 210.42 & $62.9 \%$ & $37.1 \%$ & 542773.09 & 412.96 \\
\hline Kurklareli & 390.41 & 215.49 & $64.4 \%$ & $35.6 \%$ & 593626.45 & 422.90 \\
\hline Çanakkale & 521.36 & 218.02 & $70.5 \%$ & $29.5 \%$ & 792750.48 & 427.87 \\
\hline Balıkesir & 390.41 & 218.02 & $64.2 \%$ & $35.8 \%$ & 593626.45 & 427.87 \\
\hline Bursa & 295.91 & 212.96 & $58.2 \%$ & $41.8 \%$ & 449940.28 & 417.93 \\
\hline Yalova & 312.34 & 215.49 & $59.2 \%$ & $40.8 \%$ & 474926.46 & 422.90 \\
\hline Kocaeli & 146.63 & 210.42 & $41.1 \%$ & $58.9 \%$ & 222961.07 & 412.96 \\
\hline Bilecik & 163.54 & 215.49 & $43.1 \%$ & $56.9 \%$ & 248663.39 & 422.90 \\
\hline Sakarya & 146.63 & 215.49 & $40.5 \%$ & $59.5 \%$ & 222961.07 & 422.90 \\
\hline
\end{tabular}

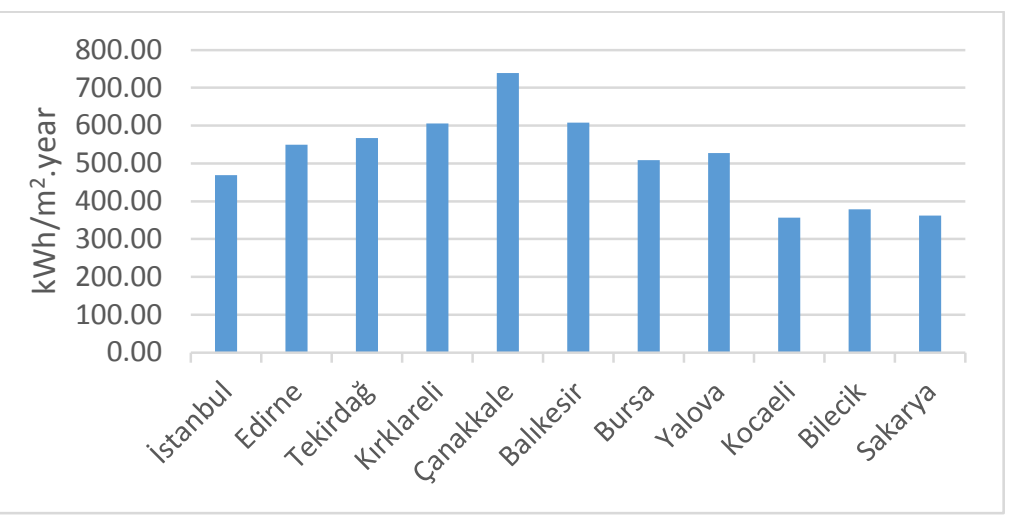

Figure 3.1: Hybrid Energy Potential of Marmara Region

For Marmara region wind energy looking very important according to calculated data. And wind energy has higher impact on hybrid energy. In this region Çanakkale have the highest hybrid energy potential. $70.5 \%$ of total energy potential comes from wind energy and $29.5 \%$ comes from sun.

Table 2.2. Wind and Solar Energy calculation for Black sea Region

\begin{tabular}{|c|c|c|c|c|c|c|}
\hline Province & $\begin{array}{c}\text { Wind } \\
\text { potential } \\
\left(\mathbf{k W h} / \mathbf{m}^{2}\right) \\
\end{array}$ & $\begin{array}{c}\text { Solar } \\
\text { Potential } \\
\left(\mathbf{k W h} / \mathbf{m}^{2}\right) \\
\end{array}$ & $\begin{array}{c}\text { Wind } \\
\text { percentage }\end{array}$ & $\begin{array}{c}\text { Solar } \\
\text { percentage }\end{array}$ & $\begin{array}{c}\text { Yearly wind } \\
\text { energy } \\
\text { (kWh/turbine) }\end{array}$ & $\begin{array}{c}\text { Yearly solar } \\
\text { energy } \\
(\mathrm{kWh} / \text { panel })\end{array}$ \\
\hline Düzce & 29.80 & 210.42 & $12.4 \%$ & $87.6 \%$ & 45310.38 & 412.96 \\
\hline Zonguldak & 68.66 & 210.42 & $24.6 \%$ & $75.4 \%$ & 104395.13 & 412.96 \\
\hline Bolu & 146.63 & 215.49 & $40.5 \%$ & $59.5 \%$ & 222961.07 & 422.90 \\
\hline Karabük & 97.00 & 210.42 & $31.6 \%$ & $68.4 \%$ & 147494.74 & 412.96 \\
\hline Bartın & 97.00 & 210.42 & $31.6 \%$ & $68.4 \%$ & 147494.74 & 412.96 \\
\hline Kastamonu & 163.54 & 215.49 & $43.1 \%$ & $56.9 \%$ & 248663.39 & 422.90 \\
\hline Çorum & 122.19 & 220.55 & $35.7 \%$ & $64.3 \%$ & 185800.89 & 432.84 \\
\hline Sinop & 190.79 & 210.42 & $47.6 \%$ & $52.4 \%$ & 290107.29 & 412.96 \\
\hline Samsun & 211.97 & 215.49 & $49.6 \%$ & $50.4 \%$ & 322313.53 & 422.90 \\
\hline Amasya & 163.54 & 215.49 & $43.1 \%$ & $56.9 \%$ & 248663.39 & 422.90 \\
\hline Tokat & 234.67 & 210.42 & $52.7 \%$ & $47.3 \%$ & 356819.28 & 412.96 \\
\hline Ordu & 201.14 & 205.36 & $49.5 \%$ & $50.5 \%$ & 305845.09 & 403.02 \\
\hline Giresun & 149.91 & 207.89 & $41.9 \%$ & $58.1 \%$ & 227941.44 & 407.99 \\
\hline Gümüşhane & 181.69 & 218.02 & $45.5 \%$ & $54.5 \%$ & 276268.74 & 427.87 \\
\hline Trabzon & 52.88 & 205.36 & $20.5 \%$ & $79.5 \%$ & 80410.83 & 403.02 \\
\hline Bayburt & 136.28 & 225.62 & $37.7 \%$ & $62.3 \%$ & 207219.49 & 442.78 \\
\hline Rize & 136.28 & 220.55 & $38.2 \%$ & $61.8 \%$ & 207219.49 & 432.84 \\
\hline Artvin & 181.69 & 225.62 & $44.6 \%$ & $55.4 \%$ & 276268.74 & 442.78 \\
\hline
\end{tabular}




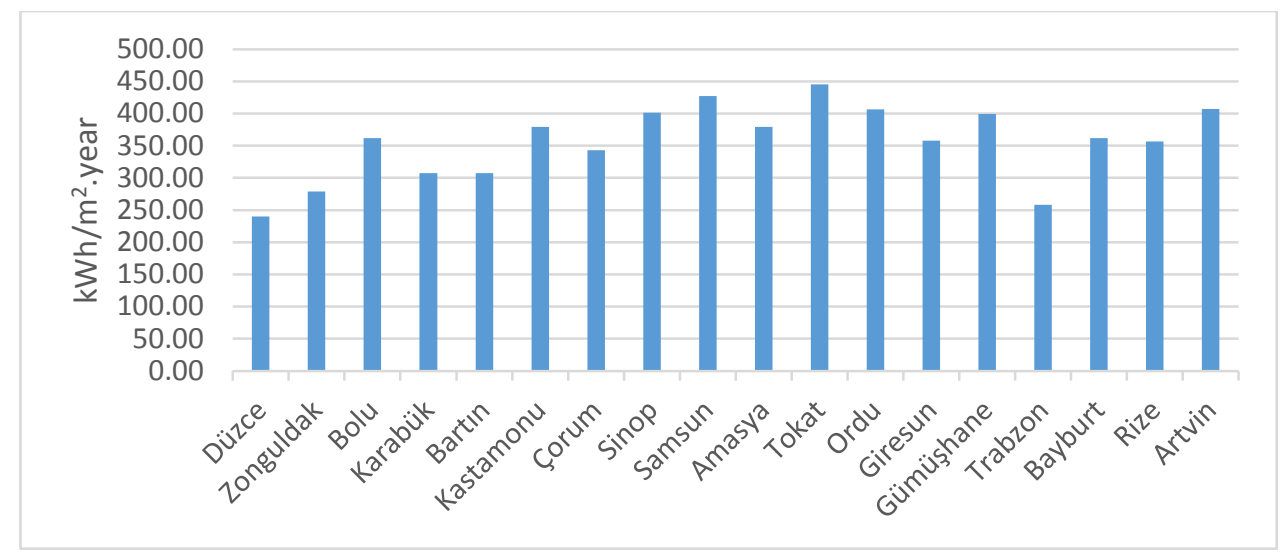

Figure 3.2: Hybrid Energy Potential of Black sea Region

In Black sea region there are 18 provinces. But region is not have much hybrid energy potential as Marmara Region. Black sea region is the north side of Turkey. So solar energy hasn't got sufficient amount of potential. Also wind energy isn't looking promising in this region but hybrid energy can be use as local purposes.

Table 2.3. Wind and Solar Energy calculation for Aegean Region

\begin{tabular}{|l|c|c|c|c|c|c|}
\hline \multicolumn{1}{|c|}{ Province } & $\begin{array}{c}\text { Wind } \\
\text { potential } \\
\left(\mathbf{k W h} / \mathbf{m}^{2}\right)\end{array}$ & $\begin{array}{c}\text { Solar } \\
\text { Potential } \\
\left(\mathbf{k W h} / \mathbf{m}^{2}\right)\end{array}$ & $\begin{array}{c}\text { Wind } \\
\text { percentage }\end{array}$ & $\begin{array}{c}\text { Solar } \\
\text { percentage }\end{array}$ & $\begin{array}{c}\text { Yearly wind } \\
\text { energy } \\
(\mathbf{k W h} / \text { turbine })\end{array}$ & $\begin{array}{c}\text { Yearly solar } \\
\text { energy } \\
(\mathbf{k W h} / \text { panel })\end{array}$ \\
\hline Kütahya & 163.54 & 220.55 & $42.6 \%$ & $57.4 \%$ & 248663.39 & 432.84 \\
\hline Afyonkarahisar & 149.91 & 243.34 & $38.1 \%$ & $61.9 \%$ & 227941.44 & 477.56 \\
\hline Uşak & 122.19 & 225.62 & $35.1 \%$ & $64.9 \%$ & 185800.90 & 442.78 \\
\hline Denizli & 97.00 & 261.07 & $27.1 \%$ & $72.9 \%$ & 147494.75 & 512.35 \\
\hline Muğla & 190.79 & 266.13 & $41.8 \%$ & $58.2 \%$ & 290107.28 & 522.29 \\
\hline Aydın & 146.63 & 250.94 & $36.9 \%$ & $63.1 \%$ & 222961.08 & 492.47 \\
\hline İzmir & 356.96 & 240.81 & $59.7 \%$ & $40.3 \%$ & 542773.08 & 472.59 \\
\hline Manisa & 211.97 & 220.55 & $49.0 \%$ & $51.0 \%$ & 322313.54 & 432.84 \\
\hline
\end{tabular}

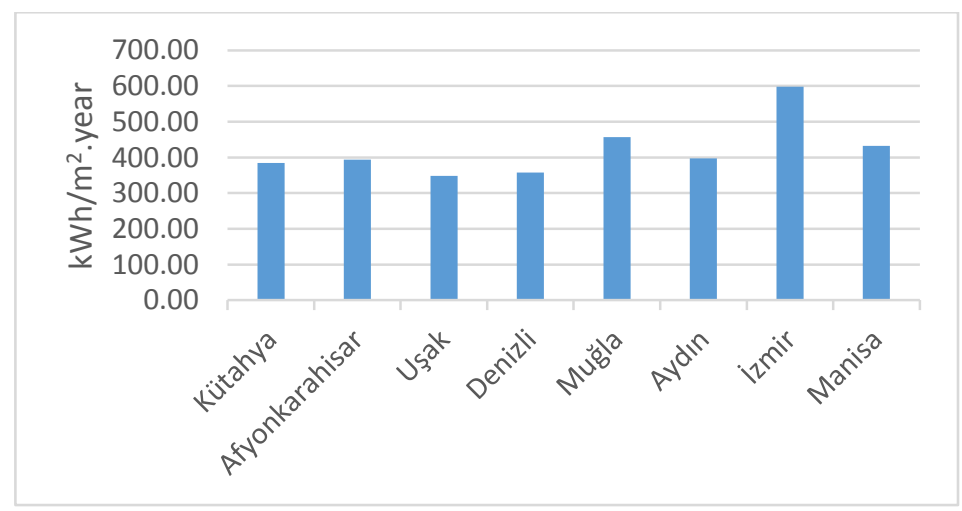

Figure 3.3: Hybrid Energy Potential of Aegean Region

For Aegean region hybrid energy looking good when considering Turkey. But in İzmir both wind and solar energy potential looking good for hybrid energy. 59.7\% of total energy potential comes from wind energy and rest of $40.3 \%$ comes from solar energy. This region may be good spot for hybrid power plant. 
International Journal of Engineering Research And Advanced Technology, Vol.5, Issue 6, June-2019

Table 2.4. Wind and Solar Energy calculation for Central Anatolia Region

\begin{tabular}{|l|c|c|c|c|c|c|}
\hline Province & $\begin{array}{c}\text { Wind } \\
\text { potential } \\
\left(\mathbf{k W h} / \mathbf{m}^{2}\right)\end{array}$ & $\begin{array}{c}\text { Solar } \\
\text { Potential } \\
\left(\mathbf{k W h} / \mathbf{m}^{2}\right)\end{array}$ & $\begin{array}{c}\text { Wind } \\
\text { percentage }\end{array}$ & $\begin{array}{c}\text { Solar } \\
\text { percentage }\end{array}$ & $\begin{array}{c}\text { Yearly wind } \\
\text { energy } \\
\mathbf{k W h} / \text { turbine) }\end{array}$ & $\begin{array}{c}\text { Yearly solar } \\
\text { energy } \\
(\mathbf{k W h} / \mathbf{p a n e l})\end{array}$ \\
\hline Ankara & 134.41 & 235.75 & $36.3 \%$ & $63.7 \%$ & 204380.98 & 462.65 \\
\hline Eskişehir & 109.11 & 225.62 & $32.6 \%$ & $67.4 \%$ & 165911.52 & 442.78 \\
\hline Çankır1 & 211.97 & 225.62 & $48.4 \%$ & $51.6 \%$ & 322313.54 & 442.78 \\
\hline Kırıkkale & 77.60 & 220.55 & $26.0 \%$ & $74.0 \%$ & 117995.79 & 432.84 \\
\hline Kırşehir & 77.60 & 228.15 & $25.4 \%$ & $74.6 \%$ & 117995.79 & 447.74 \\
\hline Konya & 284.80 & 248.41 & $53.4 \%$ & $46.6 \%$ & 433046.44 & 487.50 \\
\hline Aksaray & 122.19 & 235.75 & $34.1 \%$ & $65.9 \%$ & 185800.90 & 462.65 \\
\hline Nevşehir & 163.54 & 230.68 & $41.5 \%$ & $58.5 \%$ & 248663.39 & 452.71 \\
\hline Yozgat & 181.69 & 225.62 & $44.6 \%$ & $55.4 \%$ & 276268.75 & 442.78 \\
\hline Kayseri & 390.41 & 243.34 & $61.6 \%$ & $38.4 \%$ & 593626.45 & 477.56 \\
\hline Sivas & 312.34 & 233.21 & $57.3 \%$ & $42.7 \%$ & 474926.45 & 457.68 \\
\hline Niğde & 190.79 & 235.75 & $44.7 \%$ & $55.3 \%$ & 290107.28 & 462.65 \\
\hline Karaman & 425.88 & 250.94 & $62.9 \%$ & $37.1 \%$ & 647560.91 & 492.47 \\
\hline
\end{tabular}

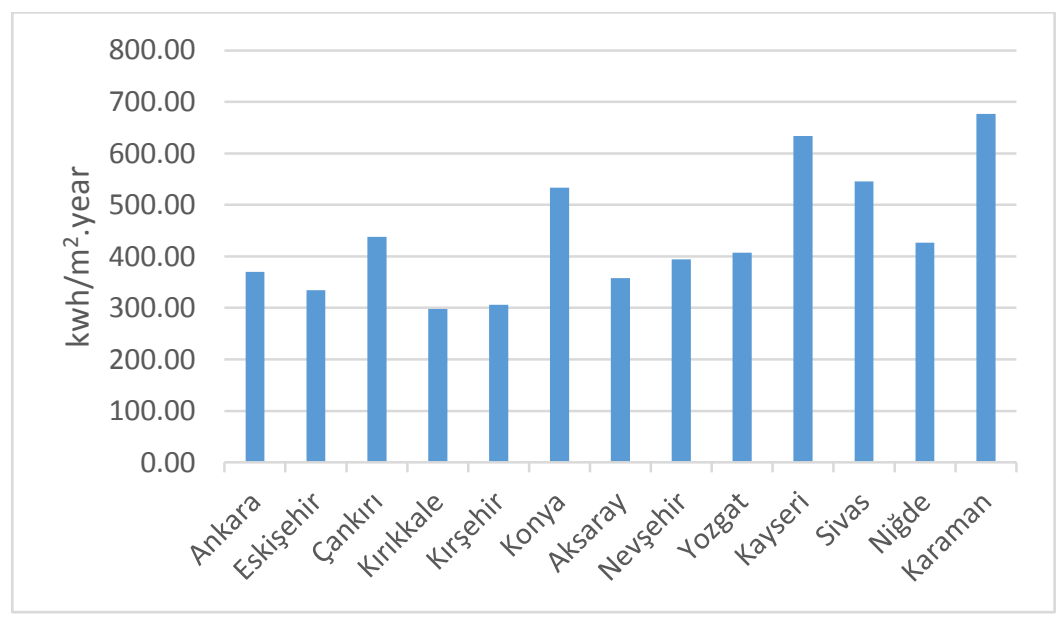

Figure 3.4: Hybrid Energy Potential of Central Anatolia Region

After the calculations that made for central Anatolian region south side of central Anatolia has higher hybrid energy potential than north side. In this region Karaman province has the highest hybrid energy potential. Karaman has 3rd highest potential in Turkey. South side of this region has great potential of hybrid energy.

Table 2.5. Wind and Solar Energy calculation for Mediterranean Region

\begin{tabular}{|l|c|c|c|c|c|c|}
\hline \multicolumn{1}{|c|}{ Province } & $\begin{array}{c}\text { Wind } \\
\text { potential } \\
\left(\mathbf{k W h} / \mathbf{m}^{2}\right)\end{array}$ & $\begin{array}{c}\text { Solar } \\
\text { Potential } \\
\left(\mathbf{k W h} / \mathbf{m}^{2}\right)\end{array}$ & $\begin{array}{c}\text { Wind } \\
\text { percentage }\end{array}$ & $\begin{array}{c}\text { Solar } \\
\text { percentage }\end{array}$ & $\begin{array}{c}\text { Yearly generated } \\
\text { wind energy } \\
(\mathbf{k W h} / \text { turbine })\end{array}$ & $\begin{array}{c}\text { Yearly generated } \\
\text { solar energy } \\
(\mathbf{k W h} / \mathbf{p a n e l})\end{array}$ \\
\hline Burdur & 146.63 & 261.07 & $36.0 \%$ & $64.0 \%$ & 222961.08 & 512.35 \\
\hline Isparta & 181.69 & 253.47 & $41.8 \%$ & $58.2 \%$ & 276268.75 & 497.44 \\
\hline Antalya & 163.54 & 261.07 & $38.5 \%$ & $61.5 \%$ & 248663.39 & 512.35 \\
\hline Mersin & 258.93 & 258.54 & $50.0 \%$ & $50.0 \%$ & 393703.81 & 507.38 \\
\hline Adana & 312.34 & 243.34 & $56.2 \%$ & $43.8 \%$ & 474926.45 & 477.56 \\
\hline Kahramanmaraş & 211.97 & 248.41 & $46.0 \%$ & $54.0 \%$ & 322313.54 & 487.50 \\
\hline Osmaniye & 258.93 & 245.88 & $51.3 \%$ & $48.7 \%$ & 393703.81 & 482.53 \\
\hline Hatay & 579.29 & 248.41 & $70.0 \%$ & $30.0 \%$ & 880833.86 & 487.50 \\
\hline
\end{tabular}




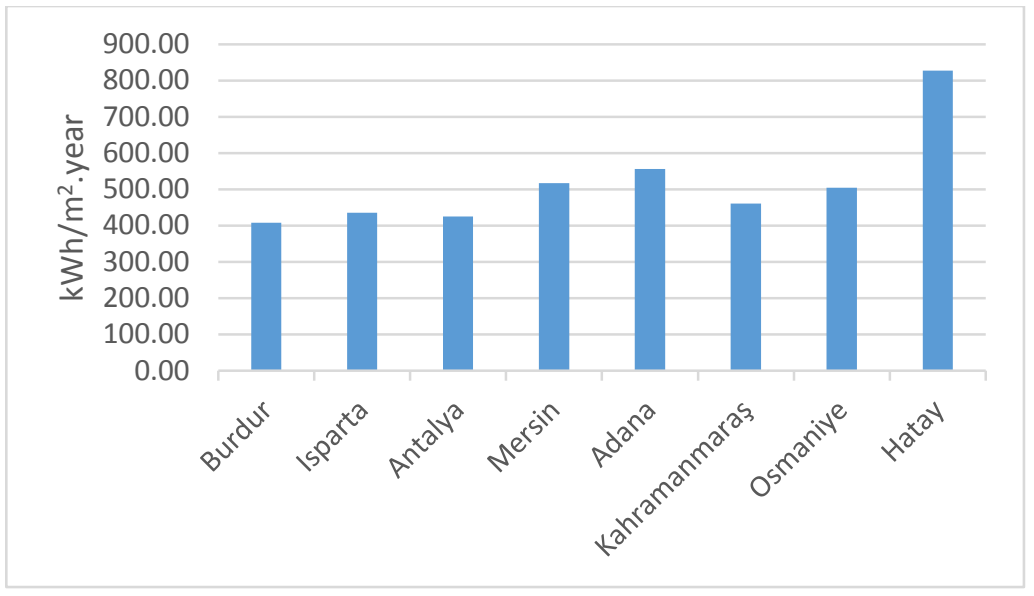

Figure 3.5: Hybrid Energy Potential of Mediterranean Region

In Mediterranean region solar radiation is highest of Turkey. Also wind energy is one of the important source of this region especially at Hatay. Hatay has great amount of wind potential and good amount of solar energy potential. According the data in table $2.570 \%$ of energy comes from wind energy and 30\% of energy potential comes from solar energy. Hatay has the highest hybrid energy potential according to calculated data. So this region has important potential for hybrid energy.

Table 2.6. Wind and Solar Energy calculation for East Anatolia Region

\begin{tabular}{|c|c|c|c|c|c|c|}
\hline Province & $\begin{array}{c}\text { Wind } \\
\text { potential } \\
\left(\mathbf{k W h} / \mathbf{m}^{2}\right)\end{array}$ & $\begin{array}{c}\text { Solar } \\
\text { Potential } \\
\left(\mathbf{k W h} / \mathbf{m}^{2}\right)\end{array}$ & $\begin{array}{c}\text { Wind } \\
\text { percentage }\end{array}$ & $\begin{array}{c}\text { Solar } \\
\text { percentage }\end{array}$ & $\begin{array}{l}\text { Yearly generated } \\
\text { wind energy } \\
\text { (kWh/turbine) }\end{array}$ & $\begin{array}{l}\text { Yearly generated } \\
\text { solar energy } \\
\text { (kWh/panel) }\end{array}$ \\
\hline Ardahan & 166.55 & 235.75 & $41.4 \%$ & $58.6 \%$ & 253246.34 & 462.65 \\
\hline Kars & 181.69 & 238.28 & $43.3 \%$ & $56.7 \%$ & 276268.75 & 467.62 \\
\hline Iğdır & 258.93 & 210.42 & $55.2 \%$ & $44.8 \%$ & 393703.81 & 412.96 \\
\hline Ağrn & 258.93 & 210.42 & $55.2 \%$ & $44.8 \%$ & 393703.81 & 412.96 \\
\hline Van & 284.80 & 291.45 & $49.4 \%$ & $50.6 \%$ & 433046.44 & 571.98 \\
\hline Hakkari & 136.28 & 291.45 & $31.9 \%$ & $68.1 \%$ & 207219.49 & 571.98 \\
\hline Bitlis & 258.93 & 293.99 & $46.8 \%$ & $53.2 \%$ & 393703.81 & 576.95 \\
\hline Muş & 149.91 & 293.99 & $33.8 \%$ & $66.2 \%$ & 227941.44 & 576.95 \\
\hline Erzurum & 312.34 & 225.62 & $58.1 \%$ & $41.9 \%$ & 474926.45 & 442.78 \\
\hline Erzincan & 312.34 & 238.28 & $56.7 \%$ & $43.3 \%$ & 474926.45 & 467.62 \\
\hline Tunceli & 221.94 & 230.68 & $49.0 \%$ & $51.0 \%$ & 337460.41 & 452.71 \\
\hline Bingöl & 149.91 & 240.81 & $38.4 \%$ & $61.6 \%$ & 227941.44 & 472.59 \\
\hline Elazı̆ & 234.67 & 240.81 & $49.4 \%$ & $50.6 \%$ & 356819.28 & 472.59 \\
\hline Malaty & 258.93 & 245.88 & $51.3 \%$ & $48.7 \%$ & 393703.81 & 482.53 \\
\hline
\end{tabular}

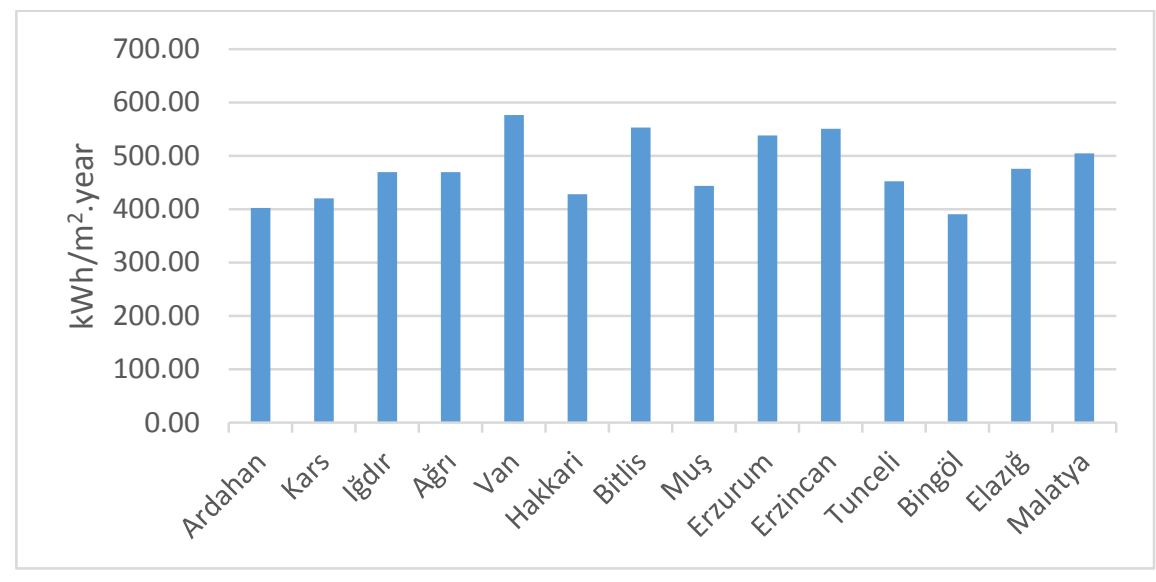

Figure 3.6: Hybrid Energy Potential of East Anatolia Region

East Anatolia Region has good potential for hybrid energy. Solar energy portion is higher than wind energy portion in some provinces. Van has good amount of hybrid energy potential. According to data $49.4 \%$ of potential comes from wind energy and $50.6 \%$ of potential comes from solar energy. 
International Journal of Engineering Research And Advanced Technology, Vol.5, Issue 6, June-2019

Table 2.7. Wind and Solar Energy calculation for South East Anatolia Region

\begin{tabular}{|l|c|c|c|c|c|c|}
\hline \multicolumn{1}{|c|}{ Province } & $\begin{array}{c}\text { Wind } \\
\text { potential } \\
\left(\mathbf{k W h} / \mathbf{m}^{2}\right)\end{array}$ & $\begin{array}{c}\text { Solar } \\
\text { Potential } \\
\left(\mathbf{k W h} / \mathbf{m}^{2}\right)\end{array}$ & $\begin{array}{c}\text { Wind } \\
\text { percentage }\end{array}$ & $\begin{array}{c}\text { Solar } \\
\text { percentage }\end{array}$ & $\begin{array}{c}\text { Yearly generated } \\
\text { wind energy } \\
\text { (kWh/turbine) }\end{array}$ & $\begin{array}{c}\text { Yearly generated } \\
\text { solar energy } \\
(\mathbf{k W h} / \mathbf{p a n e l})\end{array}$ \\
\hline Şırnak & 134.41 & 245.88 & $35.3 \%$ & $64.7 \%$ & 204380.98 & 482.53 \\
\hline Siirt & 97.00 & 245.88 & $28.3 \%$ & $71.7 \%$ & 147494.75 & 482.53 \\
\hline Batman & 134.41 & 248.41 & $35.1 \%$ & $64.9 \%$ & 204380.98 & 487.50 \\
\hline Mardin & 163.54 & 250.94 & $39.5 \%$ & $60.5 \%$ & 248663.39 & 492.47 \\
\hline Diyarbakır & 163.54 & 245.88 & $39.9 \%$ & $60.1 \%$ & 248663.39 & 482.53 \\
\hline Şanlıurfa & 60.43 & 256.00 & $19.1 \%$ & $80.9 \%$ & 91881.91 & 502.41 \\
\hline Adiyaman & 134.41 & 250.94 & $34.9 \%$ & $65.1 \%$ & 204380.98 & 492.47 \\
\hline Gaziantep & 163.54 & 250.94 & $39.5 \%$ & $60.5 \%$ & 248663.39 & 492.47 \\
\hline Kilis & 109.11 & 248.41 & $30.5 \%$ & $69.5 \%$ & 165911.52 & 487.50 \\
\hline
\end{tabular}

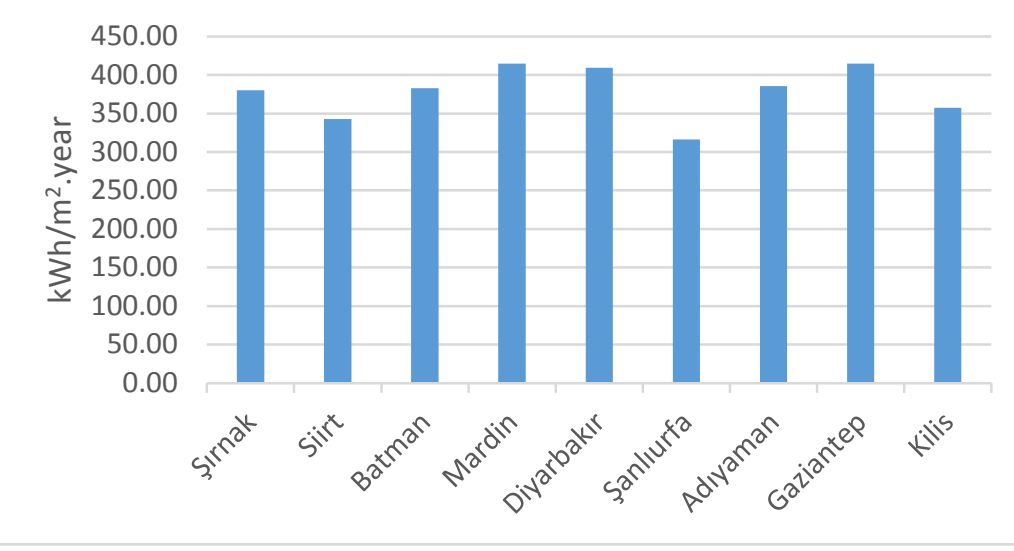

Figure 3.7: Hybrid Energy Potential of South East Anatolia Region

After the calculations for south east Anatolia region, it is found that region has good solar energy potential. But wind energy potential is not sufficient for this region according the data. Total hybrid energy potential is nearly below of country average. Low wind energy potential affects the total hybrid energy potential amount. So region has lower hybrid energy potential.

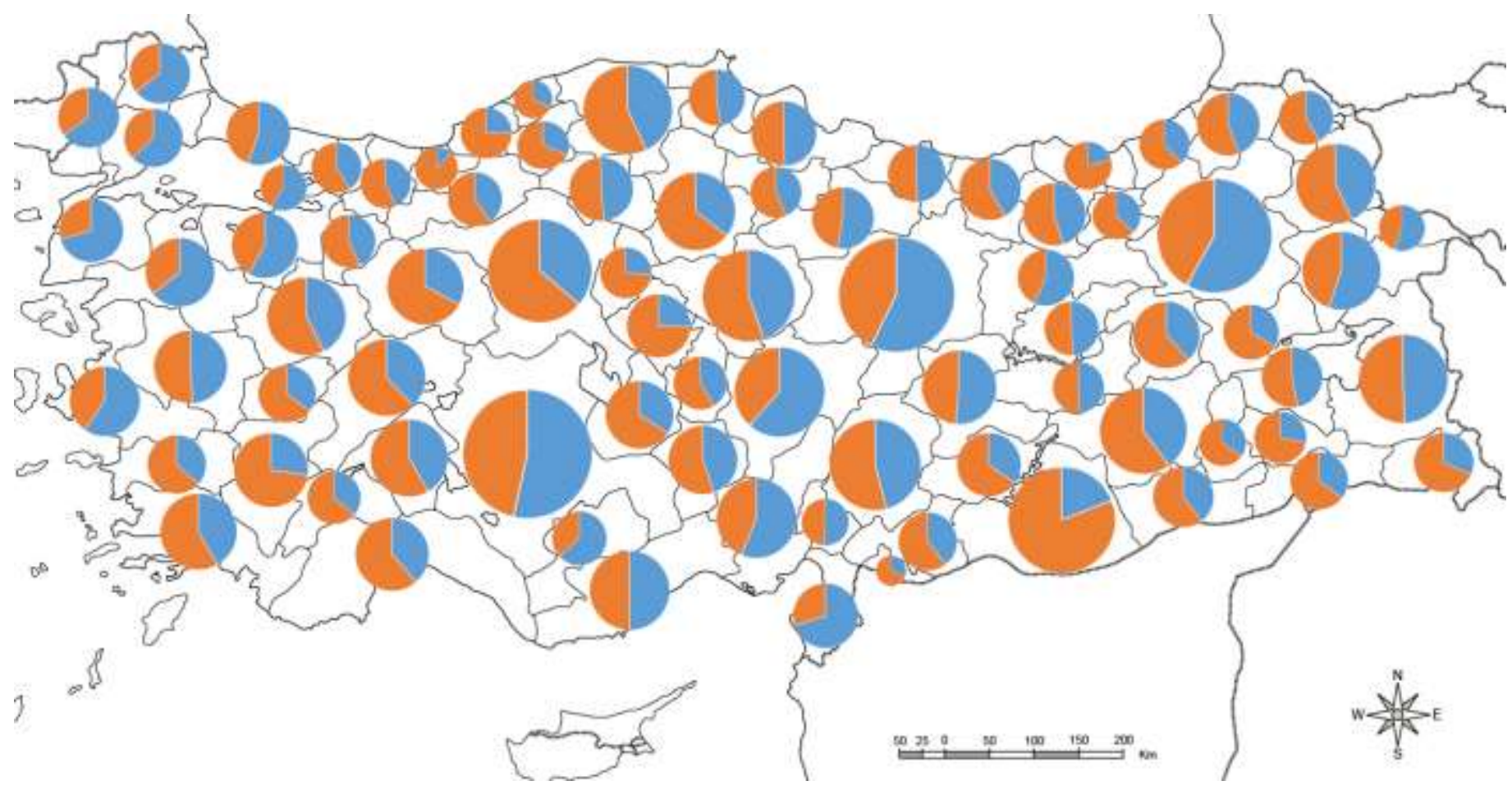

Figure 3.8: Hybrid Energy Potential Map of Turkey 
In figure 3.8 Hybrid energy potential of Turkey is given according to calculated data. Where orange color shows solar energy potential and blue color shows wind energy potential. Dimensions of graphs for each province arranged according to province area. It does not show potential amount.

\subsection{Cost Analysis Data}

Data has been calculated as mentioned before was placed in tables. $1 \mathrm{MW}$ hybrid power plant combined with wind turbine and photovoltaic solar panel with corresponding amount of potential. Wind power plant net present worth and solar power plant net present worth combined and $1 \mathrm{MW}$ hybrid power plant net present worth calculated for 25 years lifetime. Turkey divided to 7 regions. And in these regions there are 81 provinces. For each provinces $1 \mathrm{MW}$ hybrid power plant scenario has been applied. Hybrid power plant location selected be using best hybrid energy potential zone. According the potential data unit cost of electricity has been calculated for each province.

Table 3.8. 1 MW Hybrid Power Plant cost analysis for Marmara Region

\begin{tabular}{|l|c|c|c|c|}
\hline \multicolumn{1}{|c|}{ Province } & Wind Npw & Solar Npw & Hybrid Npw & $\begin{array}{c}\text { Unit cost of } \\
\text { electiricity (\$/kWh) }\end{array}$ \\
\hline İstanbul & -100857.71 & 272925.96 & 172068.26 & 0.02397 \\
\hline Edirne & 459839.83 & 130089.67 & 589929.51 & 0.01722 \\
\hline Tekirdağ & 414204.03 & 185995.47 & 600199.50 & 0.01734 \\
\hline Kurklareli & 584218.59 & 178529.75 & 762748.34 & 0.01577 \\
\hline Çanakkale & 1148820.45 & 146453.51 & 1295273.96 & 0.01279 \\
\hline Balıkesir & 577621.31 & 186450.38 & 764071.68 & 0.01579 \\
\hline Bursa & 83354.41 & 245091.76 & 328446.18 & 0.02105 \\
\hline Yalova & 163721.31 & 238976.72 & 402698.03 & 0.01994 \\
\hline Kocaeli & -287845.61 & 363558.13 & 75712.52 & 0.02834 \\
\hline Bilecik & -222672.18 & 355577.14 & 132904.96 & 0.02631 \\
\hline Sakarya & -293152.50 & 386074.68 & 92922.19 & 0.02804 \\
\hline
\end{tabular}

Table 3.9. 1 MW Hybrid Power Plant cost analysis for Black sea Region

\begin{tabular}{|l|c|c|c|c|}
\hline \multicolumn{1}{|c|}{ Province } & Wind Npw & Solar Npw & Hybrid Npw & $\begin{array}{c}\text { Unit cost of } \\
\text { electiricity (\$/kWh) }\end{array}$ \\
\hline Düzce & -143533.97 & 583815.00 & 440281.03 & 0.01937 \\
\hline Zonguldak & -227220.56 & 480746.13 & 253525.57 & 0.02480 \\
\hline Bolu & -293152.50 & 386074.68 & 92922.19 & 0.02804 \\
\hline Karabük & -307612.78 & 436529.17 & 128916.38 & 0.02844 \\
\hline Bartın & -307612.78 & 436529.17 & 128916.38 & 0.02844 \\
\hline Kastamonu & -222672.18 & 355577.14 & 132904.96 & 0.02631 \\
\hline Çorum & -392790.60 & 459089.53 & 66298.93 & 0.03013 \\
\hline Sinop & -262410.43 & 324564.61 & 62154.18 & 0.02746 \\
\hline Samsun & -171753.24 & 315339.46 & 143586.22 & 0.02516 \\
\hline Amasya & -222672.18 & 355577.14 & 132904.96 & 0.02631 \\
\hline Tokat & -54489.92 & 266550.47 & 212060.55 & 0.02317 \\
\hline Ordu & -206960.42 & 289287.40 & 82326.98 & 0.02650 \\
\hline Giresun & -271527.66 & 346396.62 & 74868.97 & 0.02813 \\
\hline Gümüşhane & -313702.23 & 370203.62 & 56501.39 & 0.02817 \\
\hline Trabzon & -265412.71 & 505535.65 & 240122.94 & 0.02575 \\
\hline Bayburt & -343920.12 & 452335.55 & 108415.43 & 0.02841 \\
\hline Rize & -339330.43 & 429212.05 & 89881.63 & 0.02876 \\
\hline Artvin & -156581.37 & 368705.86 & 212124.50 & 0.02419 \\
\hline
\end{tabular}

Table 3.10. 1 MW Hybrid Power Plant cost analysis for Aegean Region

\begin{tabular}{|l|c|c|c|c|}
\hline \multicolumn{1}{|c|}{ Province } & Wind Npw & Solar Npw & Hybrid Npw & $\begin{array}{c}\text { Unit cost of } \\
\text { electiricity (\$/kWh) }\end{array}$ \\
\hline Kütahya & -228534.89 & 377623.32 & 149088.43 & 0.02608 \\
\hline Afyonkarahisar & -307206.26 & 505256.18 & 198049.92 & 0.02610 \\
\hline Uşak & -396787.73 & 482714.47 & 85926.74 & 0.02971 \\
\hline Denizli & -334891.20 & 683520.91 & 348629.71 & 0.02397 \\
\hline Muğla & -165287.61 & 528864.42 & 363576.81 & 0.02205 \\
\hline Aydın & -326515.06 & 546894.26 & 220379.19 & 0.02592 \\
\hline
\end{tabular}


International Journal of Engineering Research And Advanced Technology, Vol.5, Issue 6, June-2019

\begin{tabular}{|l|c|c|c|c|}
\hline İzmir & 342267.73 & 287162.70 & 629430.42 & 0.01744 \\
\hline Manisa & -179508.92 & 335783.59 & 156274.68 & 0.02505 \\
\hline
\end{tabular}

Table 3.11. 1 MW Hybrid Power Plant cost analysis for Central Anatolia Region

\begin{tabular}{|l|c|c|c|c|}
\hline \multicolumn{1}{|c|}{ Province } & Wind Npw & Solar Npw & Hybrid Npw & $\begin{array}{c}\text { Unit cost of } \\
\text { electiricity (\$/kWh) }\end{array}$ \\
\hline Ankara & -359740.67 & 502917.56 & 143176.88 & 0.02787 \\
\hline Eskişehir & -276348.19 & 479419.05 & 203070.86 & 0.02617 \\
\hline Çankırı & -187085.10 & 356379.66 & 169294.56 & 0.02492 \\
\hline Kırıkkale & -373207.13 & 538492.85 & 165285.72 & 0.02859 \\
\hline Kırşehir & -376369.33 & 576328.29 & 199958.96 & 0.02775 \\
\hline Konya & 124434.47 & 353182.99 & 477617.46 & 0.01915 \\
\hline Aksaray & -237615.49 & 496456.65 & 258841.16 & 0.02450 \\
\hline Nevşehir & -239808.39 & 422098.10 & 182289.71 & 0.02560 \\
\hline Yozgat & -156581.35 & 368705.86 & 212124.51 & 0.02419 \\
\hline Kayseri & 514548.02 & 268110.57 & 782658.59 & 0.01590 \\
\hline Sivas & 125880.31 & 301479.25 & 427359.56 & 0.01994 \\
\hline Niğde & -129524.15 & 397084.40 & 267560.24 & 0.02307 \\
\hline Karaman & 687689.85 & 265989.08 & 953678.93 & 0.01453 \\
\hline
\end{tabular}

Table 3.12. 1 MW Hybrid Power Plant cost analysis for Mediterranean Region

\begin{tabular}{|l|c|c|c|c|}
\hline \multicolumn{1}{|c|}{ Province } & Wind Npw & Solar Npw & Hybrid Npw & $\begin{array}{c}\text { Unit cost of } \\
\text { electiricity (\$/kWh) }\end{array}$ \\
\hline Burdur & -334981.58 & 593744.57 & 258762.99 & 0.02532 \\
\hline Isparta & -189272.08 & 489582.44 & 300310.36 & 0.02315 \\
\hline Antalya & -270401.80 & 558253.49 & 287851.69 & 0.02414 \\
\hline Mersin & -17718.81 & 424879.67 & 407160.86 & 0.02067 \\
\hline Adana & 105340.79 & 338112.26 & 443453.06 & 0.01991 \\
\hline Kahramanmaraş & -219115.23 & 450807.58 & 231692.35 & 0.02432 \\
\hline Osmaniye & 2757.81 & 374689.56 & 377447.36 & 0.02084 \\
\hline Hatay & 1387059.96 & 201189.62 & 1588249.58 & 0.01155 \\
\hline
\end{tabular}

Table 3.13. 1 MW Hybrid Power Plant cost analysis for East Anatolia Region

\begin{tabular}{|l|c|c|c|c|}
\hline \multicolumn{1}{|c|}{ Province } & Wind Npw & Solar Npw & Hybrid Npw & $\begin{array}{c}\text { Unit cost of } \\
\text { electiricity } \mathbf{( \$ / k W h )}\end{array}$ \\
\hline Ardahan & -232809.05 & 438955.73 & 206146.68 & 0.02510 \\
\hline Kars & -171978.34 & 423194.82 & 251216.48 & 0.02372 \\
\hline Iğdır & -100857.73 & 272925.97 & 172068.24 & 0.02397 \\
\hline Ağrı & -100857.73 & 272925.97 & 172068.24 & 0.02397 \\
\hline Van & 52827.59 & 522148.31 & 574975.89 & 0.01870 \\
\hline Hakkari & -226868.72 & 727516.19 & 500647.47 & 0.02046 \\
\hline Bitlis & -70064.38 & 569634.09 & 499569.71 & 0.02008 \\
\hline Muş & -181462.85 & 706769.62 & 525306.77 & 0.01975 \\
\hline Erzurum & 141792.52 & 274434.04 & 416226.57 & 0.01995 \\
\hline Erzincan & 115516.10 & 319715.82 & 435231.92 & 0.01993 \\
\hline Tunceli & -148387.86 & 363012.67 & 214624.82 & 0.02393 \\
\hline Bingö1 & -304872.50 & 493727.36 & 188854.86 & 0.02625 \\
\hline Elazı̆ & -104314.52 & 386430.66 & 282116.14 & 0.02269 \\
\hline Malatya & 2757.81 & 374689.56 & 377447.36 & 0.02084 \\
\hline
\end{tabular}

Table 3.14. 1 MW Hybrid Power Plant cost analysis for South East Anatolia Region

\begin{tabular}{|l|c|c|c|c|}
\hline \multicolumn{1}{|c|}{ Province } & Wind Npw & Solar Npw & Hybrid Npw & $\begin{array}{c}\text { Unit cost of } \\
\text { electiricity } \mathbf{( \$ / k W h )}\end{array}$ \\
\hline Şırnak & -367932.54 & 550000.30 & 182067.76 & 0.02717 \\
\hline Siirt & -327553.78 & 608707.33 & 281153.55 & 0.02520 \\
\hline Batman & -369912.78 & 561828.31 & 191915.53 & 0.02700 \\
\hline Mardin & -260702.41 & 512446.55 & 251744.14 & 0.02463 \\
\hline
\end{tabular}




\begin{tabular}{|l|c|c|c|c|}
\hline Diyarbakır & -255672.75 & 489695.39 & 234022.64 & 0.02487 \\
\hline Şanlıurfa & -260938.28 & 739167.65 & 478229.37 & 0.02068 \\
\hline Adıyaman & -205039.75 & 539883.43 & 334843.68 & 0.02275 \\
\hline Gaziantep & -260702.41 & 512446.55 & 251744.14 & 0.02463 \\
\hline Kilis & -290635.25 & 588862.81 & 298227.56 & 0.02439 \\
\hline
\end{tabular}

After the calculation it has seen wind power side of hybrid power plant not looking profitable for some provinces. This is because Turkish government incentives for solar energy is much higher than wind energy. Also wind turbine is more expensive when compared the sufficient amount of solar panel. Wind power is much higher at $100 \mathrm{~m}$ above of the ground. Because of that wind turbine tower must be longer. This increases the wind turbine prices. Even wind power plant may not be profitable for itself it is profitable for total hybrid power plant. Also when the night time wind energy can be generated with this hybrid system. This provides continuity for energy supply which is crucial for energy demand.

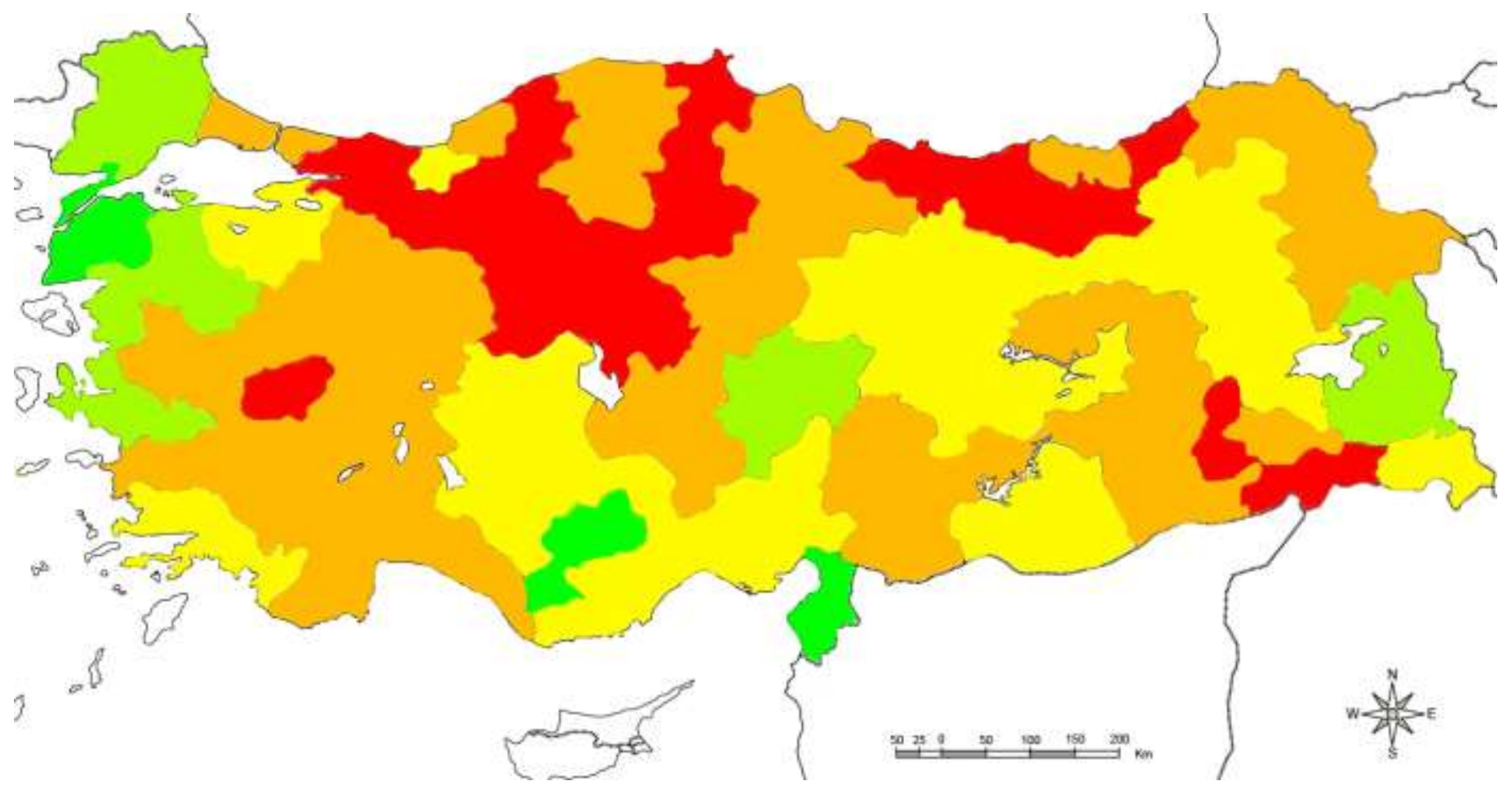

Figure 3.9: Hybrid Energy unit cost of electricity map of Turkey

In figure 3.9 unit cost of electricity has shown for each province by using colors. Dark green color representing values between 0.01342 and $0.01777(\$ / \mathrm{kWh})$, light green color representing values between 0.02212 and $0.02647(\$ / \mathrm{kWh})$, yellow color represents values between 0.02647 and $0.03082(\$ / \mathrm{kWh})$, orange color representing values between 0.03082 and 0.03517 $(\$ / \mathrm{kWh})$.

\section{CONCLUSION}

In this study Turkey's wind and solar potential has been calculated. According to calculation Turkey has great potential for hybrid energy. For 7 regions and 81 provinces of Turkey potential data has been found and according to data hybrid power plant economic scenario have been analyzed. With regard to scenario $1 \mathrm{MW}$ Hybrid power plant constructed for each 81 provinces on the best hybrid energy potential zone. According to results Hatay is the best possible location for hybrid power plant for Turkey. Then Çanakkale and Karaman follows Hatay. These provinces have some common characteristic. One of them is wind energy potential. Also these provinces have good amount of solar energy potential. The other provinces may not be high as Hatay, Çanakkale and Karaman but they have good hybrid energy potential too. Due to its local applicability, easy commissioning and high production capacity, the hybrid power plant can be used as a very convenient source of energy for many provinces. It is the responsibility of every citizen to reduce their carbon footprint by using a clean energy source. Otherwise, the Earth will continue to become increasingly uninhabitable. Hybrid energy can be the clean and renewable alternative source. Referring to Turkey as compared to the overall cost analysis was found to be a sufficient level for hybrid energy investment. So hybrid energy is not only clean and sustainable, at the same time this system makes it lucrative to be applied as energy solutions in Turkey. 


\section{REFERANCES}

[1] Republic of Turkey Ministry of Energy and Natural Sources, Electric Generation https://www.enerji.gov.tr/trTR/Sayfalar/Elektrik

[2] Perrera F. "Pollution from Fossil-Fuel Combustion is the Leading Environmental Threat to Global Pediatric Health and Equity: Solutions Exist”, Int J Environ Res Public Health. 2017 Dec 23;15(1). pii: E16

[3] Uğurlu A, Gokcol C. “An overview of Turkey's renewable energy trend,” Journal of Energy Systems 2017; 1(4):148-158.

[4] Republic of Turkey Ministry of Energy and Natural Sources, Wind Energy Data, http://www.yegm.gov.tr/yenilenebilir/ruzgarruzgar_enerjisi.aspx

[5] Republic of Turkey Ministry of Energy and Natural Sources, Solar Energy Data, http://www.yegm.gov.tr/yenilenebilir/g_enj_tekno.aspx

[6] Republic of Turkey Ministry of Energy and Natural Sources, Solar Energy, https://www.enerji.gov.tr/en-US/Pages/Solar

[7] A.B. Kanase-Patil, R.P. Saini, M.P. Sharma, "Integrated renewable energy systems for off grid rural electrification of remote area", Renewable Energy 35 2010, pp. 1342 -1349.

[8] Rajoriya, E. Fernandez, "Sustainable energy generation using hybrid energy system for remote hilly rural area in India", International Journal of Sustainable Engineering, May 2010, pp.1-9.

[9] Ajai Gupta, R.P. Saini, M.P. Sharma, "Steady-state modelling of hybrid energy system for off grid electrification of cluster of villages", Renewable Energy 35, 2010, pp. 520 - 535.

[10] Ahmad Agus Setiawan, Yu Zhao, Chem. V. Nayar, "Design, economic analysis and environmental considerations of minigrid hybrid power system with reverse osmosis desalination plant for remote areas", Renewable Energy 34,2009, pp. 374-383.

[11] Akella A.K., Sharma M.P. and Saini R.P., "Optimum utilization of renewable energy sources in a remote area," Renewable and Sustainable Energy Reviews 11, 2007, pp. 894-908.

[12] W. D. Kellogg, M. H. Nehrir, G. Venkataramanan, and V. Greez, "Generating Unit Sizing and Cost Analysis for Stand-alone Wind, Photovoltaic and Hybrid Wind/PV Systems," IEEE Trans. Energy Conversion, Vol. 13, No. 1, pp. 70-75, March 1998.

[13] Kurozumi, Kazuhiro et al, "Hybrid system composed of a wind power and a photovoltaic system at NTT Kume-jima radio relay station,” INTELEC, International Telecommunications Energy Conference 1998, pp. 785-789.

[14] Riad Chedid and Saifur Rahman, "Unit Sizing and Control of Hybrid Wind-Solar Power Systems," IEEE Trans. Energy Conversion, Vol. 12, No. 1, pp. 79-85, March 1997.

[15] Francois Giraud and Zyiad M. Salameh, "Steady-State Performance of a Grid-Connected Rooftop Hybrid Wind-Photovoltaic Power System with Battery Storage,” IEEE Trans. Energy Conversion, Vol. 16, No. 1, pp. 1- 7, March 2001

[16] Jie Li et.al, "Feasibility analysis of applying the wind-solar hybrid generation system in pastoral area," Mongolia University of science \& technology (2012).

[17] Renewable Energy General Management, Wind energy potential map of Turkey, http://www.yegm.gov.tr/YEKrepa/REPAduyuru_01.html

[18] Republic of Turkey Ministry of Agriculture and Forestry, Meteorological Service, Yearly global solar radiation map of Turkey, https://www.mgm.gov.tr/kurumici/radyasyon_iller.aspx

[19] Kaya, K., Koç, E. 2015. “Enerji Üretim Santralleri Maliyet Analizi,” Mühendis ve Makina, issue 660, pp. 61-68.

[20] 29/12/2010 dated law no: 6094, Government incentive prices, http://www.mevzuat.gov.tr/MevzuatMetin/1.5.5346.pdf

[21] Republic of Turkey Energy Market Regulatory Authority, Electric Price, https://www.epdk.org.tr/Detay/Icerik/3-

1327/elektrik-faturalarina-esas-tarife-tablolari 